\title{
La hormonoterapia de reemplazo disminuye la mortalidad aún en mujeres con historia familiar de cáncer de mama
}

The role of hormone replacement therapy in the risk for breast cancer and total mortality in women with a family history of breast cancer. Thomas AS, Pamela JM; James RC et al. Ann Intern Med. 1997;127:973-980.

\section{Objetivo}

Determinar el tipo de asociación entre terapia de reemplazo hormonal (TRH), cáncer de mama y mortalidad total en mujeres posmenopaúsicas con antecedentes familiares de cáncer de mama.

\section{Diseño}

Estudio de cohorte prospectivo con seguimiento a 8 años.

\section{Lugar}

Iowa. Estados Unidos.

\section{Participantes}

A partir de una muestra randomizada de 41837 participantes del "Estudio para la Salud de la Mujer en Iowa" de 1985, se seleccionaron 35919 mujeres entre 55 y 69 años. Criterios de exclusión: premenopausia, mastectomía previa, historia de cualquier cáncer diferente del de piel e historia familiar no conocida de cáncer de mama.

\section{Evaluación de factores pronósticos}

Las participantes respondieron cuestionarios autoadministrados que incluían: datos personales sobre educación, hábitos, medidas antropométricas, condiciones clínicas específicas (diabetes, enfermedad cardíaca), historia ginecológica, antecedentes familiares de cáncer de mama, consumo de hormonas especificando duración ( $\leq 1$ mes, 2-6 meses, 7-12 meses, 13-24 meses, 3-5 años y > 5 años) e historia de mamografías realizadas.

\section{Medición de resultados}

Se categorizó la TRH según uso actual, uso previo o nunca expuestas; y por duración en $\leq 5$ ó > 5 años. Se evaluó la asociación entre historia familiar y TRH con la incidencia de cáncer de mama y mortalidad total ajustando por edad y otras variables clínicas relevantes.

\section{Resultados Principales}

El 12.2\% de la cohorte tenía antecedentes familiares de cáncer mamario. La incidencia de cáncer de mama en las mujeres que recibieron TRH por al menos 5 años fue de $61 \times 10000$ personas-año (IC95\% 28-94); en las que nunca habían recibido TRH la incidencia fue de $46 \times 10000$ personas-año (95\% IC 36-55). Este aumento no fue estadísticamente significativo. En las mujeres con historia familiar positiva, la mortalidad total fue menor en las que habían recibido TRH en comparación con las nunca reemplazadas: RR 0.67 (IC95\% 0.51-0.89). Incluyendo cualquier tipo de cáncer el RR fue 0.75 (IC95\% 0.5-1.12). Ajustando por edad, la mortalidad anual para mujeres que usaban TRH por al menos 5 años fue la mitad comparada con las que nunca habían utilizado: 45 muertes / 10000 personas-año (IC95\% 19-74) vs. $80 / 10000$ personas-año (IC95\% 69-92 muertes) respectivamente.

\section{Conclusiones}

En mujeres con antecedentes familiares de cáncer mamario la TRH aumentó de manera no significativa la incidencia de cáncer de mama. Sin embargo disminuyó significativamente la mortalidad total. Esto se traduciría en un balance beneficioso de la intervención.

\section{COMENTARIO}

Este trabajo nos enfrenta con los riesgos y beneficios de la TRH tomando en cuenta el cáncer de mama y la mortalidad total. Plantea la problemática en cuanto a la recomendación y consejo sobre TRH. Entre los factores de riesgo conocidos para cáncer de mama, es importante el antecedente familiar de esta enfermedad, particularmente cuando se trata de la madre o hermana (RR 1,5-2); y a su vez los riesgos son mayores en relación con la edad de aparición y extensión de la enfermedad. Los estudios publicados sugieren una asociación entre TRH y aumento del riesgo de cáncer de mama (RR 1,35, IC95\% 1,21-1,49) en las usuarias de más de 5 años y aún mayor en mujeres con historia familiar (RR 3,4 IC95\% $2,0-6,0)(1-3)$. El estudio de las enfermeras reportó un $45 \%$ de aumento en muertes por cáncer de mama en mujeres con más de 5 años de TRH. No obstante los estudios epidemiológicos hasta el momento sugieren que las mujeres que reciben TRH tienen menor riesgo global de muerte, explicado fundamentalmente por la disminución de eventos cardiovasculares (4). En el presente estudio no se validó fehacientemente el antecedente familiar de cáncer de mama, no se especificó la causa de indicación de la hormonoterapia ni la composición hormonal utilizada. A pesar de éstas limitaciones tiene importancia clínica ya que es clara la disminución de la mortalidad total y no tan importante el aumento en la incidencia de cáncer de mama en estas mujeres con mayor riesgo. Esto es similar a lo hallado por Grodstein y cols. en el estudio de las enfermeras, en que también se registró una reducción de la mortalidad global. Con estos datos se podría pensar y decidir en forma conjunta con la paciente el uso de TRH, evaluando el riesgo individual y conociendo que, si bien aumenta el riesgo de cáncer de mama, disminuye la mortalidad total aún en pacientes con antecedentes familiares de la enfermedad.

Dra. Vilda Discacciati 